\title{
UNA FAMILIA DE MUJERES INMIGRANTES ITALIANAS EN EL GRAN BUENOS AIRES (CA. 1950)
}

\author{
A family of Italian immigrant women in Greater Buenos Aires
}

(ca. 1950)

Denise Rocío Ganza*

\begin{abstract}
Resumen. Nuestra investigación se interesa por el influjo de la inmigración transatlántica en un espacio particular del Gran Buenos Aires (Valentín Alsina). En este contexto, decidimos abordar las trayectorias migratorias y de integración a la sociedad receptora de las integrantes de una familia de hermanas italianas que llegaron a la zona luego de 1950. A través del uso de fuentes orales, contemplamos la influencia de los condicionantes de género en las distintas etapas del proceso migratorio, en cuestiones variadas como la toma de la decisión de emigrar, los matrimonios, el trabajo y la vida familiar.
\end{abstract}

Palabras clave: inmigración italiana; género; Gran Buenos Aires; Siglo XX.

\begin{abstract}
Our research is interested in the influence of transatlantic immigration in a particular area of Greater Buenos Aires (Valentín Alsina). In this context, we decided to focus on the migratory trajectories and the integration into the host society of the members of a family of Italian sisters who arrived to the area after 1950. We used oral sources to contemplate the influence of gender conditioning factors in the different stages of the migration process, on various issues such as the decision-making to emigrate, marriages, work, and family life.
\end{abstract}

Keywords: immigration; gender; Greater Buenos Aires; Twentieth Century.

Valentín Alsina es una localidad del Gran Buenos Aires (Argentina) creada oficialmente en 1875. En la actualidad forma parte del partido de Lanús, contiguo a la Capital Federal, a la cual el área de nuestro interés se encuentra unida por un puente que atraviesa el Riachuelo, tal como puede observarse en la Figura 1.

Esta zona reúne algunas características particulares que la convierten en un espacio privilegiado para realizar un análisis microhistórico de las profundas transformaciones acontecidas en la provincia de Buenos Aires, especialmente en las zonas aledañas a la Capital Federal, desde fines del siglo XIX: los fenómenos de urbanización, industrialización y marcado incremento de la población de

Instituto de Investigaciones Gino Germani y Facultad de Filosofía y Letras, Universidad de Buenos Aires. Buenos Aires, Argentina. E-mail: deniseganza@yahoo.com.ar. Orcid: https://orcid.org/00000001-5994-5824. 
origen extranjero. En este sentido, Valentín Alsina se ha caracterizado, en primer lugar, por el importante componente inmigratorio de su población, expresado en la diversidad de colectivos nacionales asentados en la zona y la vitalidad de sus formas asociativas. En segundo término, la localidad se convirtió en un importante enclave industrial. Finalmente, no podemos dejar de señalar la escasa atención historiográfica de la que ha gozado, no solo este espacio en particular sino el conjunto del Gran Buenos Aires, hasta la fecha.

\section{Figura 1 - Ubicación geográfica de Valentín Alsina}

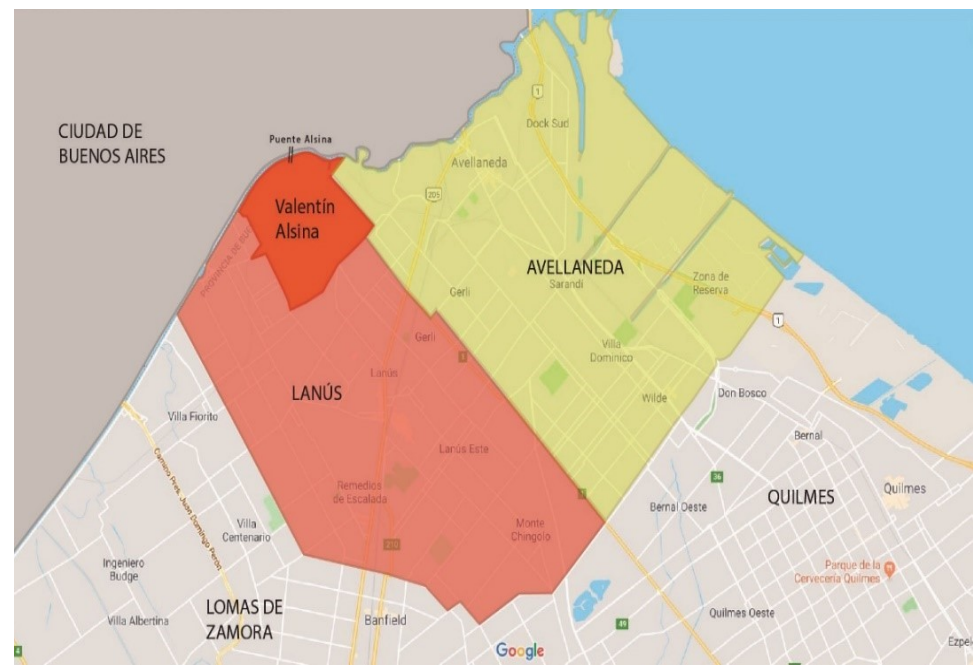

Fuente: Elaboración propia (en colaboración con la Diseñadora Gráfica Romina Costa) sobre la base de < https://www.google.com/maps>. Consulta: 10.12.18.

En esta ocasión, nos proponemos específicamente abordar las trayectorias personales de las integrantes de una familia de hermanas provenientes de Polla (Italia), con la intención de conocer las particularidades de las experiencias migratorias y de integración en la sociedad receptora de los oriundos de Italia, a través de un ejercicio de reducción de la escala analítica. A propósito de ello, es importante recordar brevemente que la colectividad italiana revistió una gran importancia en la zona. Los censos nacionales de 1895 y 1914 mostraron su preponderancia a nivel municipal ${ }^{1}$ hacia fines del siglo XIX, aunque también su sustitución por el grupo español, en tanto primer colectivo inmigrante en importancia, en la ocasión posterior (Poder Ejecutivo Nacional, 1898; Poder Ejecutivo Nacional, 1916). No obstante, el periodo de interés de este trabajo se inicia con la finalización de la Segunda Guerra Mundial, momento a partir del cual se

\footnotetext{
Es preciso aclarar que hasta la autonomía de Lanús, efectuada en el año 1944, todo el territorio de este partido, incluida la localidad de Valentín Alsina, se encontraba comprendido en el municipio de Avellaneda (anteriormente denominado Barracas al Sud).
} 
evidenció una recuperación de los flujos migratorios desde Italia hacia la Argentina. Esta situación se relacionó fundamentalmente con el rápido restablecimiento de la economía del país austral, simultáneo respecto de la implementación de restricciones a la inmigración en otros destinos preferenciales tales como Estados Unidos, Canadá, Australia y Nueva Zelanda. Por otra parte, la recuperación de los flujos se produjo incluso a pesar de los intentos de control establecidos también en la Argentina (Devoto, 2006, p. 328-331; Bjerg, 2009, p. 28-29).

En nuestro caso, la consideración de otras fuentes, tales como los registros parroquiales y los Libros de desembarco del puerto de Buenos Aires, nos permitió evidenciar los efectos de esta oleada inmigratoria y la centralidad del arribo de italianos que implicó, con su consecuente impacto en cuanto al asentamiento de individuos provenientes de Italia en Valentín Alsina (Parroquia San Juan Bautista, 1926-1935, 1946-1955; Centro De Estudios Migratorios Latinoamericanos, s./f.). En particular, además, pudimos constatar la importancia de la región de Campania y la provincia de Salerno, en las que se ubica el lugar de origen de las inmigrantes de nuestro interés, como espacios de partida (Parroquia San Juan Bautista, 1926-1935, 1946-1955; Centro De Estudios Migratorios Latinoamericanos, s./f.).

Con el fin de comprender las trayectorias migratorias de estas mujeres, a lo largo de este artículo nos valdremos de dos estrategias metodológicas fundamentales: el uso de fuentes orales y la aplicación de una perspectiva de género, que nos permita observar los condicionantes atravesados por las hermanas Sessa en los distintos momentos del proceso migratorio, como la toma de la decisión de emigrar, los matrimonios, el trabajo y la vida familiar.

Por lo tanto, en el primer apartado de este escrito nos ocuparemos de realizar un balance acerca del impacto de la utilización del concepto de género en el campo de los estudios migratorios y profundizar en la descripción e implicancias del tipo de fuentes utilizadas. Luego, a lo largo de los tres apartados siguientes, nos detendremos en el relato de las trayectorias de estas mujeres migrantes, dividiendo su experiencia según las etapas de pre-migración, migración y pos-migración. Finalmente, realizaremos algunas reflexiones a modo de conclusión.

\section{Estudios migratorios, perspectiva de género y uso de fuentes orales}

La perspectiva de género comenzó a abrirse paso en la historiografía a partir de la década de 1960, principalmente en el mundo anglosajón (Barrancos, 1993). En relación con los aportes en esta materia dentro del campo de los estudios migratorios, es preciso señalar que los abordajes acerca de los condicionantes impuestos por el género en el marco de las experiencias migratorias han cobrado un interés creciente a lo largo de la última década.

Entendemos por "género", junto con Scott (2008), la organización social de la diferencia sexual. Se trata de un sistema estructurador presente en las sociedades humanas, que implica relaciones de poder (Gregorio Gil, 1997; Scott, 
2008). Sus discursos suelen operar como mecanismos de control informales (Silva García, 2019). Sin embargo, resulta pasible de ser transformado ante coyunturas o fenómenos sociales de distinto tipo (Rosas, 2010). Es imprescindible enfatizar en su carácter relacional, puesto que afecta tanto a varones como a mujeres, en la medida que ambos se encuentran atravesados por las normas y tradiciones construidas por la sociedad (Rosas, 2010). No obstante, frecuentemente, la perspectiva de género ha sido asociada a la historia de las mujeres, la cual trata de subsanar la falta de conocimiento acerca de aspectos fundamentales de su participación como sujetos sociales (McGee Deutsch, 2017).

Puntualmente, los procesos migratorios pueden ser considerados una de aquellas coyunturas o fenómenos que son capaces de "introducir transformaciones o adaptaciones temporales en las situaciones y relaciones de género de los y las migrantes" (Rosas, 2010, p. 17). Sin embargo, como ya dijéramos, la importancia de este último aspecto en la bibliografía acerca de las migraciones ha ido creciendo paulatinamente. En un balance al respecto, Gregorio Gil (1997) demuestra que tradicionalmente la migración femenina no era siquiera considerada una emigración laboral, sino que se la interpretaba exclusivamente dentro del marco de su rol en el ámbito doméstico. Luego, algunos trabajos pioneros establecieron una diferenciación entre las causas atribuidas a la migración masculina (económicas) y la migración femenina (sociales), caracterizando a esta última por el deseo de independencia familiar y social. No obstante, otras aproximaciones no tardaron en reposicionar los flujos de mujeres dentro de la esfera de lo económico y productivo. Finalmente, en las últimas décadas, la consideración de la migración como una estrategia puesta en práctica por los grupos domésticos y su concreción a través del funcionamiento de redes sociales habilitó el análisis de las desigualdades de género como uno de los elementos que se juegan en las interacciones dentro de los miembros de estos grupos.

Para analizar nuestro caso, y a los fines de organizar la exposición, retomaremos la propuesta de Grieco y Boyd (s./f.), para quienes la influencia del género puede evaluarse en las tres etapas del proceso migratorio: pre-migración, migración y pos-migración.

Por otra parte, es importante detenernos en las características de la aproximación metodológica elegida en materia de fuentes, basada en los aportes de la Historia Oral. Al respecto, creemos preciso enfatizar que este procedimiento de estudio resulta especialmente útil en el marco de nuestra investigación, en tanto permite acercarse a "los procesos sociales y la vida de la gente 'común'" (Benadiba, Plotinsky, 2007, p. 10) y "esclarecer experiencia humana subjetiva" (Taylor, Bogdan, 1992, p. 106). En este sentido, hemos partido de la premisa propuesta por Bartolucci y Favero (2008) acerca de que las fuentes orales no deben ser consideradas solamente como material complementario de otras fuentes tradicionales, sino que se trata de testimonios valiosos por sí mismos, capaces de ofrecer información completa sobre la dimensión subjetiva del proceso migratorio. 
Es decir, resultan significativas para obtener la visión de los propios protagonistas sobre determinados sucesos, teniendo en cuenta que "las interpretaciones que los sujetos realizan sobre un determinado fenómeno son constitutivas del mismo y la metodología cualitativa es la adecuada para conocerlas" (Rosas, 2010, p. 32).

En definitiva, los testimonios personales serán el insumo fundamental de este trabajo, combinados en ocasiones con un álbum fotográfico familiar disponible y, por supuesto, con la bibliografía necesaria para ofrecer una contextualización. A continuación, el detalle sobre la construcción de las fuentes orales utilizadas.

En primer lugar, nos valimos de un grupo focal efectuado conjuntamente a las cinco hermanas Sessa. La metodología del grupo focal implicó la reunión en conjunto, a los fines de obtener datos a partir del intercambio entre estas mujeres acerca de la temática de la emigración (Barbour, 2013; Bonilla Landaverry, 2019; Kvale, 2011; Petracci, 2007). Con respecto a la elección puntual de esta herramienta para el análisis cualitativo, tuvimos en cuenta diversas cuestiones, que nos llevaron a privilegiarla por sobre la opción de realizar entrevistas individuales. En principio, el grupo focal nos pareció propicio para abordar las trayectorias de estas mujeres que, al mismo tiempo que tenían en común pertenecer a una única familia, podían disponer de experiencias variadas (Barbour, 2013) en función de sus subjetividades particulares, pasibles de ser observadas a partir de su interacción. Aspecto, este último, en el que los grupos focales han sido distinguidos de las entrevistas grupales (Petracci, 2007). Asimismo, nos pareció de mayor interés no adoptar un rol excesivamente directivo a lo largo del diálogo (Kvale, 2011) y, en cambio, privilegiar el intercambio suscitado entre las participantes, a partir de la consigna general de explorar sus trayectorias migratorias.

Para complementar el grupo focal y precisar algunos datos también realizamos una entrevista semiestructurada presencial de final abierto a María Sessa, la mayor de la familia, quien puso también a nuestra disposición el álbum fotográfico al que ya nos referimos. En esta ocasión, utilizamos una guía de temas claves, en tanto poseíamos un cierto grado de conocimiento previo sobre la persona a entrevistar (Taylor, Bogdan, 1992). Finalmente, utilizamos aquí algunos pasajes de la entrevista semiestructurada presencial de final abierto realizada a Cinthya Sacco, nieta de María, con la finalidad de observar algunas cuestiones en el plano intergeneracional.

En este punto, es importante señalar que todas las entrevistas se realizaron con el previo consentimiento de las entrevistadas, quienes tuvieron conocimiento de que estaban brindando información para un trabajo académico. Asimismo, prestaron su autorización para la divulgación de sus nombres reales, la publicación de las fotografías y la aparición de citas textuales de sus relatos. Por último, a su debido momento, tendrán acceso a los resultados de la investigación, por ejemplo al presente artículo científico. 


\section{Pre-migración: la vida en la sociedad de origen}

La historia de la familia de nuestro interés se inició con el matrimonio de Francesco Sessa y Redenta Esposito Piccolo a fines de la década de 1920. El primer hijo, y único varón, de nombre Francesco, falleció con apenas seis meses de edad. María, Elena, Giuseppa, Adelina, Ercilia y Ermelinda (que también falleció tempranamente) nacieron entre 1932 y 1945, con cuatro años como máxima diferencia entre cada nacimiento. No todas vivieron siempre en el mismo pueblo, ni arribaron conjuntamente a la ciudad de Buenos Aires, no obstante lo cual dieron lugar a la configuración familiar con la que hoy nos encontramos (Familia Sessa, grupo focal, 4 de junio de 2017; María Sessa, entrevista, 27 de noviembre de 2019).

La experiencia pre-migratoria de las hermanas Sessa estuvo marcada por dos procesos, uno de índole social y otro de índole familiar. El primero refiere a la experiencia colectiva de la Segunda Guerra Mundial. La misma fue determinante, sobre todo, para la partida de quien luego se convirtiera en el esposo de María, Antonio Sacco. El segundo se relaciona con el vínculo conflictivo entre sus padres, la posterior separación tras la contienda bélica y la dispersión de las hermanas, que vivieron en diferentes sitios hasta su reunión definitiva en territorio argentino.

Sobre la guerra, las hermanas recuerdan haberse refugiado en una gruta de varios kilómetros de extensión, en la que convivieron con otros vecinos de Polla, que recurrían a la matanza de sus animales para obtener comida. Allí estuvieron unos veinte días, refugiándose de los bombardeos. Afirman que contaban con la ayuda de los soldados alemanes, aún después del armisticio. Sobre el racionamiento, María recuerda que "Mussolini daba 100 g. de pan, 100 g. de fideos... iinegros los fideos!!" (María Sessa, entrevista, 27 de noviembre de 2019).

Pero no solo durante el conflicto las condiciones de vida fueron difíciles. Recordemos que la contienda supuso una completa alteración de la estructura económica italiana, la cual debió ser reconstruida en los años subsiguientes (Strangio, 2018).

Con respecto a la relación entre Redenta y Francesco, parece haber sido dificultosa desde el inicio mismo del matrimonio. Las hermanas se refieren a la oposición de la familia materna a este vínculo y describen a su padre como "mujeriego" y "jugador". Nunca disponían del dinero suficiente para vivir, a pesar de que Francesco solía tener buenos empleos. Asimismo, relatan que su madre tendía a culpar a su marido por la muerte del primogénito, en tanto aquél lo había llevado a otro pueblo en una carreta, tras lo cual el niño se enfermó y falleció. Después de una breve separación, Redenta y Francesco retomaron su relación, producto de la cual fueron naciendo sus hijas.

La familia atravesó por múltiples mudanzas. Por este motivo, no todas las hermanas nacieron en el mismo pueblo. María, Elena y Giuseppa lo hicieron en Teggiano; Adelina, en Albanella; y Ercilia, en Battipaglia. Todas ellas son localidades 
de la provincia de Salerno, región de Campania (Figura 2). Finalmente, la familia terminó por vivir en Polla.

Figura 2 - Ubicación geográfica de Polla y las localidades de nacimiento de las hermanas Sessa

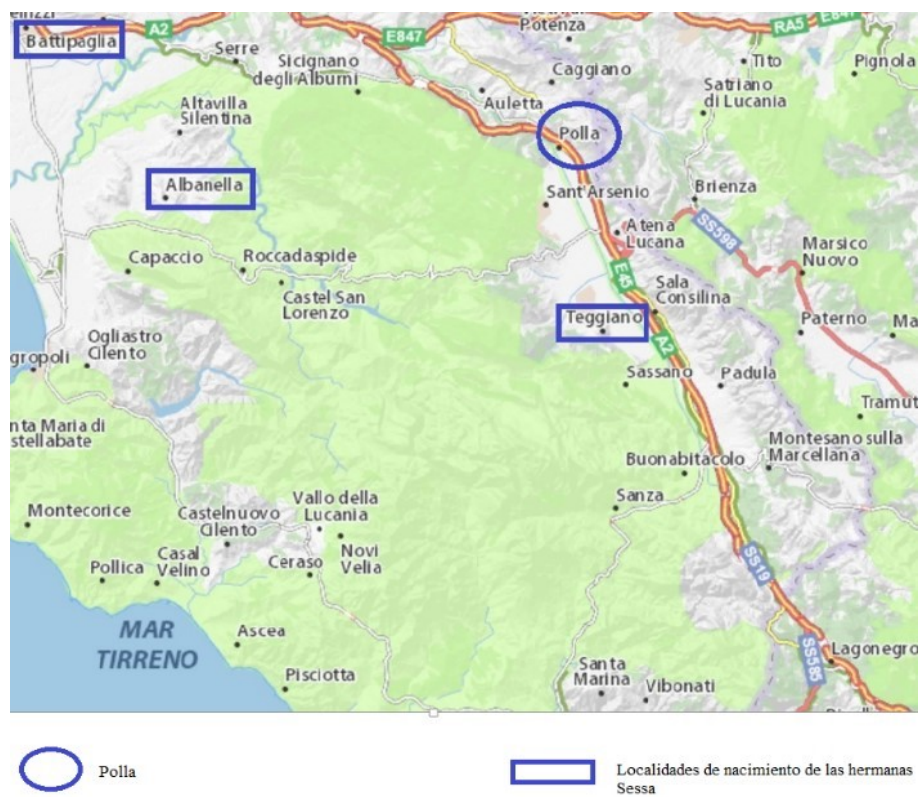

Fuente: Elaboración propia a partir del mapa disponible en < https://www.viamichelin.es/ web/Mapas-Planos/Mapa_Plano-Teggiano-84039-Salerno-Italia >. Consulta: 12.12.19.

La separación definitiva del matrimonio de Redenta y Francesco se produjo tras el fin del conflicto bélico. Él rehízo su vida en Roma, con una mujer oriunda de esa ciudad. Por su parte, Redenta tuvo que hacerle frente a largas jornadas de trabajo para sostener la economía familiar:

[...] a la mañana se levantaba e iba a una casa... la llamaba todo el pueblo... la hacían coser... le daban la máquina, le daban la tela y mi mamá cosía todo el día. Pero la gente era muy egoísta. Como dice Adelina ahora: ¿por qué mama no venía temprano? Venía a las nueve cuando ya ellas tenían frío, tenían hambre. La gente le daba la comida [...] pero ya estaban acostadas. Adelina y Ercilia fueron las que más padecieron [...]. (María Sessa, entrevista, 27 de noviembre de 2019)

En ese entonces, las hermanas ya vivían separadas. Giuseppa fue a Nápoles a trabajar en el servicio doméstico. Por su parte, María y Elena fueron a vivir con tías maternas. Según los relatos, la tía que le tocó en suerte a Elena era muy rica, por haberse casado con un empresario vinculado a las minas de carbón. El desenvolvimiento de los acontecimientos en su casa sería decisivo para la 
reunificación de toda la familia en la Argentina, hacia 1960 (Familia Sessa, grupo focal, 4 de junio de 2017).

Por todo lo anterior, podríamos sugerir que el impacto de las cuestiones de género resultó sumamente relevante para la dinámica familiar durante la etapa pre-migratoria. El alejamiento del padre y el ingreso de la madre en el mundo laboral determinaron la separación de las hermanas, aún antes de que algunas de ellas cruzaran el Atlántico.

De igual modo, no queremos dejar de señalar que, aunque no sea mencionado por las hermanas como un motivo inmediato de su partida de Italia, indudablemente los hechos de la Segunda Guerra Mundial constituyen en su relato un "hito-acontecimiento", que se convierte en una referencia de temporalidad, tal como lo consideran Bartolucci y Favero (2008). En este sentido, podríamos sostener, por ejemplo, que el propio contexto bélico habría resultado definitorio en cuanto a la separación del matrimonio de Redenta y Francesco, puesto que la misma se concretó solo en ese momento, aunque las hermanas refieren la existencia de antecedentes conflictivos en la relación desde tiempos muy tempranos.

Así como el alejamiento del padre y el ingreso de la madre en el mundo laboral fueron determinantes para la separación de las hermanas, también en lo que refiere a la decisión de emigrar, el papel de los acontecimientos familiares, el rol de la madre y la condición de mujeres de las hermanas Sessa resultaron elementos centrales.

\section{Migración: con rumbo a la Argentina}

En el caso de María y sus hermanas, el papel de los acontecimientos familiares, el rol de la madre y su condición de mujeres son elementos centrales para la decisión de emigrar.

Dos de ellas, María y Giuseppa (tercera en orden de edad), partieron de Italia luego de celebrar sus casamientos "por poder" ${ }^{2}$. Ambas refieren que, sin contar con el permiso del padre (que, de acuerdo con el relato de María, quería que se trasladaran a Roma para vivir con él), el camino de conseguir esposo en América era el único que podía garantizar la emigración (María Sessa, entrevista, 27 de noviembre de 2019). Como señala Arrieta (2018), tanto Italia como otros países europeos esbozaron "argumentos a favor de la emigración basada en el 'llamado' de un emigrante establecido en ultramar al resto de su familia o a sus paisanos" (p. 186).

María partió en primer lugar, tras contraer matrimonio con Antonio Sacco, quien ya hacía varios años que había emigrado de un pueblo cercano a Polla con destino a la Argentina, como producto de la situación económica resultante de

\footnotetext{
2 Este término hace referencia a las bodas que se celebran en presencia de uno solo de los contrayentes, mientras el otro se encuentra representado por un tercero, que cuenta con un poder especial para ello. En nuestros casos de análisis, los matrimonios se efectuaron en Italia, en presencia de las novias, mientras que los novios se encontraban residiendo en la Argentina.
} 
la Segunda Guerra Mundial. La relación de noviazgo se concretó por medio de la correspondencia, hasta que tomaron la decisión de casarse "por poder". Así lo relata María (entrevista, 27 de noviembre de 2019):

Yo me vestí de blanco, fui al civil, fui a la Iglesia, pero con mi suegro... A veces, cuando mis hijos eran chicos, decían [cuando miraban fotografías de la boda] "Pero éste no es mi papá; ¿con quién te casaste?"... Claro, era un apoderado... Así era el casamiento.

Si bien tras la ceremonia se llevó a cabo una celebración en la sociedad de origen (María Sessa, entrevista, 27 de noviembre de 2019), la Figura 3, correspondiente al álbum familiar, muestra que también se realizó una recepción en Valentín Alsina, luego de la llegada de María, que arribó al puerto de Buenos Aires el 13 de julio de 1953.

Figura 3 - Casamiento "por poder" de María Sessa (1953)

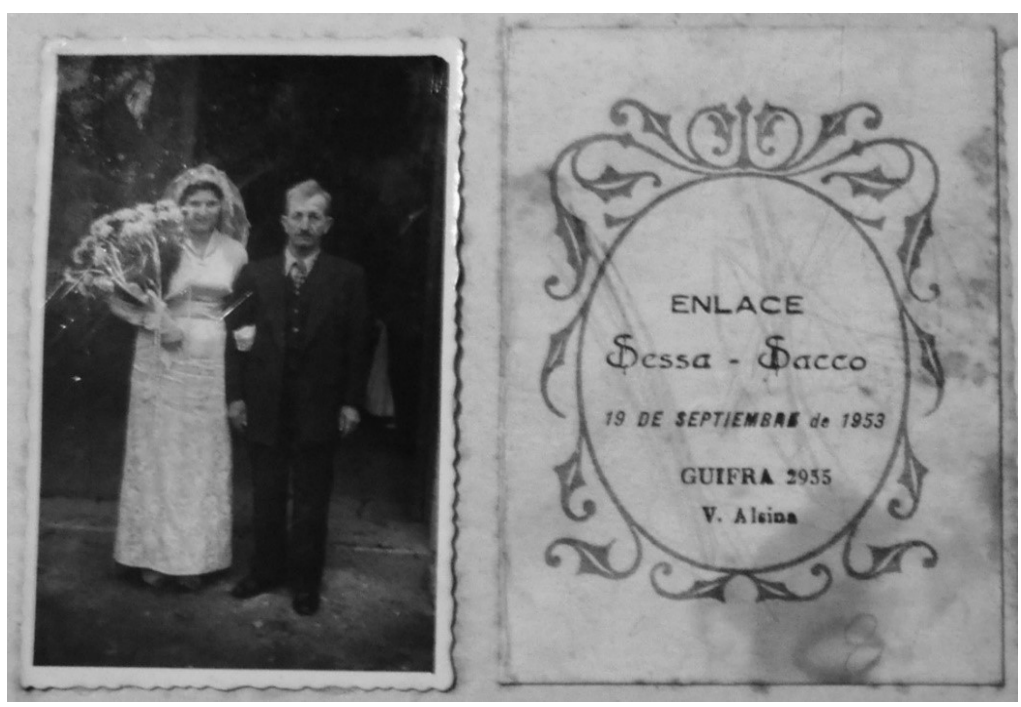

Fuente: Álbum fotográfico familiar de María Sessa.

Giuseppa, por su parte, partió del puerto de Nápoles aproximadamente un año después, a los 18 años de edad, siendo ya una mujer casada, en virtud de su propio matrimonio "por poder". Su esposo era quince años mayor que ella y la boda se había concertado por intermedio de Antonio Sacco, quien compartía vivienda en Valentín Alsina con varios hombres. Si bien algunos de ellos eran paisanos, el candidato elegido para Giuseppa fue Omar Olguín, de nacionalidad argentina.

Sin embargo, la experiencia resultó muy diferente para las dos, tanto por motivos de carácter objetivo como subjetivo. Si bien María tuvo el desafío de ser la primera de la familia en emigrar, mientras que Giuseppa ya contó con su apoyo 
al llegar a Buenos Aires, la mayor de las hermanas tuvo la ventaja de conocer a su esposo. La menor, en cambio, se vio compelida a aceptar la determinación de su madre de que partiera de Italia, donde consideraba que no tenía posibilidades de casarse por no contar con el ajuar exigido a las mujeres, y el candidato sugerido por su cuñado. De hecho, se resistió a mudarse inmediatamente con su marido, razón por la cual sostuvieron una suerte de noviazgo durante algunos meses (Familia Sessa, grupo focal, 4 de junio de 2017). Si bien ambas coinciden en afirmar que su mamá las condujo hacia la Argentina "por bronca" con su padre y para evitar que partieran a vivir con él, mientras María considera que pudo perdonar a sus progenitores, Giuseppa guarda reproches tanto hacia su madre como hacia su padre, que no logró evitar el viaje. María (entrevista, 27 de noviembre de 2019) recuerda su intervención en la concertación del matrimonio de la siguiente manera:

Yo tenía 20 años [...] Tampoco entendía mucho [...] Me escribieron de Italia, que ahí no se podía casar en el pueblo, porque necesitaba la blanquería, el ajuar, la mujer que se casaba... Nosotras no teníamos nada. La única solución era que nos viniéramos a la América.

El encuentro con las otras hermanas, que llegaron a la Argentina acompañadas por su madre, se efectuó en 1960. Según relata María, el viaje no se pagó y realizaron varios trámites en el consulado. Asimismo, durante el grupo focal, las hermanas refirieron que se trató de uno de los últimos viajes realizados a través del Comité Intergubernamental para las Migraciones Europeas (CIME) (Familia Sessa, grupo focal, 4 de junio de 2017; María Sessa, entrevista, 27 de noviembre de 2019).

Este tipo de migración asistida se inscribe dentro de las políticas de planificación instauradas por el peronismo, entre las cuales se destacaron sendos acuerdos firmados con Italia (en dos oportunidades, 1947 y 1948) y España (1948). Asimismo, la regulación de los flujos por parte del gobierno italiano incluyó otros acuerdos variados, con países e instituciones internacionales (Strangio, 2018). No obstante, la emigración asistida hacia la Argentina se vio superada ampliamente por los flujos considerados "espontáneos" (Biernat, 2007).

En particular, el acuerdo con el CIME fue suscripto en febrero de 1953. En tanto organismo internacional, aquél tenía por misión la promoción de la emigración desde Europa, facilitando los recursos necesarios a quienes no contaran con ellos. Es importante señalar que existían coincidencias fundamentales entre los objetivos del CIME y los del segundo plan quinquenal peronista, en tanto se orientaban a la reunificación familiar y la selección de trabajadores destinados a desempeñarse en el ámbito rural. Así como algunas de las hermanas Sessa, la mayor parte de los beneficiarios del CIME fueron de origen italiano, dada la temprana adhesión de Italia al convenio (en abril de 1952). 
Asimismo, es importante señalar que el CIME tuvo una intención manifiesta de favorecer los procesos de reagrupación familiar, privilegiando el viaje de mujeres y niños. De hecho, los varones de entre 18 y 50 años interesados en viajar debían realizar una contribución económica (De Cristóforis, 2016).

Sin embargo, las hermanas Sessa le otorgan una importancia central a un hecho personal atravesado por Elena, como factor decisivo en cuanto a la reunificación de la familia en la Argentina. María (entrevista, 27 de noviembre de 2019) sostiene al respecto que "en el año '60 en Italia ya estaban bien... por Elena estamos acá nosotras". Según relatan, durante la estadía en casa de la tía de mejor posición económica, Elena mantuvo un acercamiento romántico con su primo, al que la madre del muchacho se oponía, sobre todo con motivo de las diferencias económicas entre ambas familias. Por esa razón, dicha tía intentó convencer a Redenta de que partiera hacia la Argentina con Elena, Adelina y Ercilia. Inclusive, les dio una parte del dinero necesario para el viaje, con el que, al llegar a Valentín Alsina, las cuatro mujeres lograron equipar su casa (María Sessa, entrevista, 27 de noviembre de 2019).

En esta etapa de la migración propiamente dicha los condicionantes de género resultan sumamente evidentes. En primer término, podemos observar cómo el carácter de mujeres de María y Giuseppa las hizo objeto de las decisiones familiares sobre sus posibilidades. En virtud del conflicto con el padre, pero también de la falta de condiciones económicas que les permitieran afrontar un matrimonio ventajoso en la sociedad de origen, se determinó que debían emigrar. En el caso de Giuseppa, además de su madre, su hermana y, en especial un hombre, su cuñado, tuvieron un rol fundamental, concertando el matrimonio con un varón al que ella no conocía. En definitiva, nos resta decir que la propia figura del matrimonio "por poder" representaba una clara situación de vulnerabilidad para estas mujeres, que no solo abandonaban su tierra de origen sino que lo hacían con la perspectiva de encontrarse con un marido desconocido o con el que apenas habían tenido vínculo.

\section{Pos-migración: la vida en Valentín Alsina}

El primer aspecto que se destaca en el relato de la mayor de las hermanas es el de la decepción que sufrió al llegar a la Argentina y el contraste con el imaginario que circulaba en la sociedad de origen, alimentado seguramente por las corrientes de información, que hacían hincapié en las experiencias exitosas de los migrantes anteriores. En este sentido, es posible observar las diferencias existentes entre el "mundo imaginado" antes de la partida y el "mundo real", caracterizado sobre todo por las dificultades de los primeros tiempos tras el arribo al país austral (Scarzanella, 2005). Al respecto, María (entrevista, 27 de noviembre de 2019) nos relata no solo su propia impresión sino también la de su marido Antonio al llegar a Valentín Alsina: 
Creíamos que encontraríamos la plata en el piso... Cuando yo vine, la verdad, lloré mucho [...] Porque Italia es muy linda al fin y al cabo. Acá, sobre Valentín Alsina [en referencia a la calle], había piedras, pasaban los caballos con las vacas, con la leche $[\ldots]$.

Antonio [...], en la Capital, caminaba, caminaba y más o menos veía lindo, pero cuando llegó a Valentín Alsina se quería morir.

No obstante, las redes familiares y paisanales aparecen como un factor primordial de contención y una ayuda invaluable a la hora de adaptarse a la realidad del barrio suburbano. Antonio partió de Italia a los 17 años. En Valentín Alsina debía encontrarse con un tío. María refiere que sus primas lo trataron muy bien. El posterior contacto con otros hombres del mismo pueblo, pero también con nativos, posibilitó que alquilaran una casa que compartieron. Allí, como comentamos antes, Antonio conoció al que sería el futuro marido de Giuseppa (María Sessa, entrevista, 27 de noviembre de 2019).

Asimismo, sobre la experiencia de integración en la Argentina, un aspecto que se destaca especialmente es el de las cuestiones laborales. De hecho, la diferencia de menos de una década entre la llegada de María y el reencuentro de todas las hermanas en 1960 resultó sumamente significativa en cuanto al factor de inserción en este ámbito. Tampoco hay que desestimar las disimilitudes derivadas de la condición de mujeres casadas en la que llegaron María y Giuseppa, a diferencia de Elena, Adelina y Ercilia.

Las tres hermanas solteras consiguieron rápidamente trabajo. Adelina, en un comercio de la zona de Once, cuyo propietario era de origen judío. Por su parte, Elena y Ercilia se valieron de los vínculos intraétnicos para obtener un empleo en una fábrica textil de Pompeya. La madre se encargaba, mientras tanto, de las tareas domésticas (María Sessa, entrevista, 27 de noviembre de 2019).

En el caso de María y Giuseppa, su condición de mujeres casadas hizo que se dedicaran al cuidado de la casa y de los hijos, que llegaron prontamente (Familia Sessa, grupo focal, 4 de junio de 2017). María (entrevista, 27 de noviembre de 2019), además, hace referencia a las particularidades del carácter de su marido, a quien califica de "celoso" y a la condición "maleducada" y "machista" de la sociedad de acogida. Sin embargo, también considera que su insistencia en cuanto a que Antonio "le ponga un negocio" dio finalmente resultado. Su marido había tenido varios empleos en relación de dependencia y, por último, se dedicó a la comercialización de pollos y derivados en ferias barriales, como puede observarse en la Figura 4. El comercio del que se hizo cargo María fue instalado en el frente de la vivienda definitiva de la familia, alrededor de 1970, y se encargó de la provisión del mismo tipo de productos (María Sessa, entrevista, 27 de noviembre de 2019). 


\section{Figura 4 - Antonio Sacco en su puesto de comercialización de pollo y derivados (ca. 1975)}

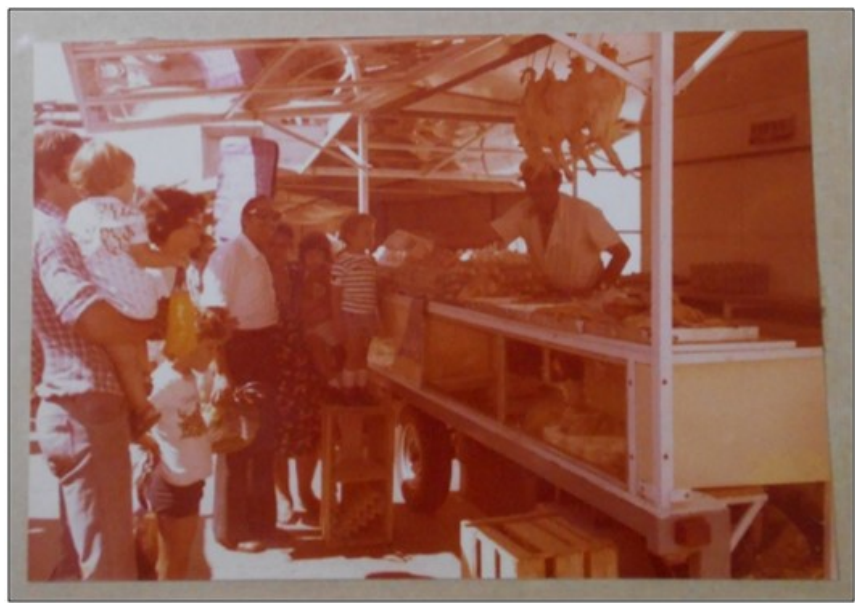

Fuente: Álbum fotográfico familiar de María Sessa.

Las diferencias señaladas anteriormente pueden ser interpretadas a partir de los aportes de dos historiadoras que se ocuparon de las cuestiones de género en la historia argentina. Por un lado, cabe recordar la afirmación de Lobato (1993) acerca de que "el proceso de conformación del ideal de domesticidad en la Argentina fue realimentado por aquellos hombres y mujeres que cruzaban el Atlántico" (p. 70). Asimismo, Wainerman (2007) se refirió a las características generales de la fuerza de trabajo femenina hasta la década de 1960. A propósito de ello, afirmó que:

[...] estaba formada predominantemente por hijas que en general salían a trabajar en su juventud, antes de casarse o de tener su primer hijo, y luego dejaban de hacerlo para dedicarse a la casa y a la crianza, porque se entendía que era parte de la hombría de bien de los maridos ser el sustento de su familia. (p. 348)

Si bien no se trata del objetivo central de este artículo, es preciso indicar que la cuestión generacional y los cambios de los condicionantes de género a lo largo del tiempo se dejaron traslucir en el relato de una de las descendientes de la familia, a quien también tuvimos ocasión de entrevistar. Cinthya Sacco, hija de Héctor, el hijo menor de María, sostuvo que:

Yo valoré mucho la vida fuera de la casa... laburaba y estudiaba... pero como que rechazaba o no me importaba aprender a tejer, a cocinar... y como que ahora me doy cuenta de que son oficios re importantes y que hubiera estado genial aprender eso de mi abuela y mis tías. (Cinthya Sacco, entrevista, 13 de diciembre de 2019)

En definitiva, para la entrevistada, las prioridades se hallaron fuera del ámbito doméstico, aunque en el presente puede valorar aquellas actividades que María y 
sus hermanas llevaban a cabo dentro del hogar, las cuales parecen haber perdido importancia entre sus hijos y nietos de ambos sexos.

En otro orden, la referencia de María a los celos de su marido, pero también a las características de la sociedad de acogida, tiene puntos de contacto con los testimonios prestados por otras mujeres inmigrantes radicadas en José C. Paz, a partir de los cuales Castiglione (2019) concluye que:

\begin{abstract}
El temor a las violaciones de la guerra, la opinión del barrio y la religiosidad se tradujeron en un cuidado excesivo de la mujer y la desconfianza de los hombres con respecto a otros; en algunos casos, se llegó a la expulsión de posibles amistades por preferir que estas fueran de su misma región o nacionalidad. Por esa razón las asociaciones, en principio, eran también espacios de encuentro para futuros matrimonios y relaciones de camaradería que consideraban confiables y seguras. (p. 341)
\end{abstract}

Asimismo, queremos recuperar brevemente la cuestión de la influencia del género en esta última etapa del proceso migratorio. A propósito de ello, destacamos sobre todo el asunto de la inserción laboral. En este punto, no solo el contexto temporal del arribo, sino también el estado civil de las mujeres se mostró como un elemento determinante. Del mismo modo, resulta notable que, por encima de las políticas migratorias favorables a nivel nacional e internacional, las hermanas ubiquen un hecho familiar y amoroso como motivo preponderante de la reunificación familiar. En la aceptación por parte de Redenta de la sugerencia de su hermana de que partieran a reunirse con María y Giuseppa, están presentes los condicionantes de género, pero también los económicos (en virtud de la diferencia social entre ambas familias), en torno a la persona de Elena.

No queremos dar por terminado este apartado sin destacar que las hermanas Sessa tuvieron la oportunidad de regresar a Italia. Paradójicamente, la única excepción fue Elena, cuyo romance fue mencionado como motivo principal de la radicación en Argentina. María, inclusive, resultó homenajeada en Polla y aprovechó la oportunidad para referirse a su madre, con quien considera que el pueblo tuvo un comportamiento injusto. Pero, además, esos viajes propiciaron cierta reconciliación familiar. María cuenta que uno de los hijos de la tía que condicionó la partida de Elena, viajó especialmente desde Venecia para verla. Además, tanto ella como Giuseppa, Adelina y Ercilia conocieron, una vez que todos los progenitores ya habían fallecido, a las hermanas que resultaron de la nueva pareja de su padre en Roma (Familia Sessa, grupo focal, 4 de junio de 2017; María Sessa, entrevista, 27 de noviembre de 2019).

\title{
Reflexiones finales
}

A lo largo de este trabajo nos propusimos ofrecer una mirada sobre la inmigración europea en Valentín Alsina que se anclara en el punto de vista personal de sus protagonistas. Así lo hicimos, por medio de la disponibilidad de testimonios 
orales, con la particularidad de que, por tratarse de mujeres migrantes, pudimos también abordar la temática desde una perspectiva de género.

En el primer apartado, entonces, detallamos el impacto de los aportes realizados en el campo de los estudios migratorios a partir de la aplicación del concepto de género y describimos las fuentes orales de las que nos valimos, con énfasis en su importancia para comprender las experiencias de los sujetos desde su propia mirada.

En cuanto a las trayectorias de las hermanas Sessa, indudablemente su experiencia migratoria muestra cómo las personas se encuentran atravesadas por las normas y tradiciones construidas por la sociedad en materia de género, tal como sostuvo Rosas (2010). Su condición de mujeres, en particular, las hizo beneficiarias de ciertas políticas migratorias a nivel nacional e internacional (especialmente las promovidas por el CIME), pero también las convirtió en objeto de las decisiones familiares y de las redes paisanales de las que participaron, donde las desigualdades de género resultaban uno de los condicionantes fundamentales de las interacciones, teniendo en cuenta que la migración se presentaba como una estrategia adoptada en conjunto por el grupo doméstico (Gregorio Gil, 1997). El acontecimiento del matrimonio "por poder" de Giuseppa se convierte, así, en un ejemplo ineludible. La decisión materna se combinó con la disponibilidad de vínculos familiares y paisanales al otro lado del Atlántico, los cuales proveyeron el candidato para el matrimonio, un hombre desconocido para la novia y quince años mayor que ella. Pero la influencia de los determinantes de género no se agota aquí; los hemos visto actuar también, junto con el elemento generacional, en cuanto a la inserción laboral.

Al inicio de este texto también nos referimos a la migración como oportunidad para la introducción de transformaciones en materia de género, aunque sea de modo temporal. En este punto, podemos concluir que para las hermanas Sessa no sucedió así. Lejos de encontrar resquicios para evadir los condicionantes de género, sus experiencias de vida parecen mostrar el notable peso que les impuso su condición de mujeres. No obstante, no podemos dejar de mencionar el rol de Redenta, la madre de las hermanas Sessa. Al respecto, hemos visto su papel decisivo para la emigración de sus hijas, sobre todo de María y Giuseppa. De hecho, si bien en muchos aspectos los condicionantes de género podían continuar presentes, esta estrategia le permitió contrarrestar las pretensiones de su ex esposo acerca de la posibilidad de que las hermanas se instalaran en Roma.

\section{Referencias bibliográficas}

ARRIETA, Sofía. "Los barcos de las esposas", La reunificación familiar en la política migratoria argentina y brasilera (1945-1950). In: MAGLIANO, María José (comp.). Entre márgenes, intersticios e intersecciones: Diálogos posibles y desafíos pendientes entre género y migraciones. Buenos Aires: Teseo, 2018, p. 171-198. Disponible en: <https://www.teseopress.com/entremargenes>. Consulta: 06.02.2020. 
BARBERO, María Inés. Estrategias de empresarios italianos en Argentina. El Grupo Devoto. Anuario CEEED, v. 1, n. 1, p. 10-42, 2009. Disponible en: <http:// www.economicas.uba.ar/wp-content/uploads/2016/03/CEEED-Estrategias-deempresarios-italianos.pdf>. Consulta: 08.06.2017.

BARBOUR, Rosaline. Los grupos de discusión en Investigación Cualitativa. Madrid: Morata, 2013.

BARRANCOS, Dora. Introducción. In: BARRANCOS, Dora (comp.). Historia y género. Buenos Aires: Centro Editor de América Latina, 1993, p. 7-12.

BARTOLUCCI, Mónica; FAVERO, Bettina. Tramas de la memoria migrante. In: FAVERO, Bettina (comp.). Voces y memoria de la inmigración: Mar del Plata en el siglo XX. Mar del Plata: EUDEM, 2008, p. 61-88.

BENADIBA, Laura; PLOTINSKY, Daniel. De entrevistadores y relatos de vida. Buenos Aires: Facultad de Filosofía y Letras, Universidad de Buenos Aires, 2007.

BIERNAT, Carolina. ¿Buenos o útiles?: La política inmigratoria del peronismo. Buenos Aires: Biblos, 2007.

BJERG, María. Historias de la inmigración en la Argentina. Buenos Aires: Edhasa, 2009.

BONILLA LANDAVERRY, Gesly Aníbal. Investigación científica: Métodos, técnicas e instrumentos. Guatemala: Serviprensa, 2019.

CASTIGLIONE, Celeste. Relatos migrantes: Historias de vida y muerte en José C. Paz. José C. Paz: Edunpaz, 2019. Disponible en: < https://edunpaz.unpaz.edu.ar/OMP/ index.php/edunpaz/catalog/book/38>. Consulta: 20.01.2019.

DE CRISTÓFORIS, Nadia Andrea. Familia y emigración en el último ciclo de las corrientes españolas hacia la Argentina (1946-1955). Odisea. Revista de Estudios Migratorios, n. 3, p. 1-29, 2016. Disponible en: < https://publicaciones.sociales. uba.ar/index.php/odisea/article/view/1932>. Consulta: 15.10.2019.

DEVOTO, Fernando. Historia de los italianos en la Argentina. Buenos Aires: Cámara de Comercio Italiana en la República Argentina, 2006.

GREGORIO GIL, Carmen. El estudio de las migraciones internacionales desde una perspectiva de género. Migraciones, v. 1, p. 145-175, 1997. Disponible en: <https://revistas.comillas.edu/index.php/revistamigraciones/article/view/4939>. Consulta: 10.10.2019.

GRIECO, Elizabeth; BOYD, Mónica. Women and Migration, incorporating gender into international migration theory. S./f. Disponible en: <http://homes.chass. utoronto.ca/ boydmon/research_papers/gender_inequality/grieco_and_boyd. pdf>. Consulta: 10.10.2019.

KVALE, Steinar. Las entrevistas en Investigación Cualitativa. Madrid: Morata, 2011.

LOBATO, Mirta Zaida. Mujeres obreras, protesta y acción gremial en la Argentina, los casos de la industria frigorífica y textil en Berisso. In: BARRANCOS, Dora (comp.). Historia y género. Buenos Aires: Centro Editor de América Latina, 1993, p. 65-97.

MCGEE DEUTSCH, Sandra. Cruzar fronteras, reclamar una nación: Historia de las mujeres judías argentinas, 1880-1955. Buenos Aires: Fondo de Cultura Económica, 2017. 
PETRACCI, Mónica. La agenda de la opinión pública a través de la discusión grupal, Una técnica de investigación cualitativa: el grupo focal. In: KORNBLIT, Ana Lía (coord.). Metodologías cualitativas en Ciencias Sociales: Modelos y procedimientos de análisis. Buenos Aires: Biblos, 2007, p. 77-89.

PODER EJECUTIVO NACIONAL. Segundo Censo de la República Argentina. Tomo II. Buenos Aires, 1898. Disponible en: <http://deie.mendoza.gov.ar/\#!/censosnacionales-de-poblacion>. Consulta: 26.02.2020.

PODER EJECUTIVO NACIONAL. Tercer Censo Nacional: levantado el $1^{\circ}$ de junio de 1914. Tomo I. Buenos Aires, 1916. Disponible en: <http://deie.mendoza.gov. ar/\#!/censos-nacionales-de-poblacion>. Consulta: 26.02.2020.

ROSAS, Carolina. Implicaciones mutuas entre el género y la migración: Mujeres y varones peruanos arribados a Buenos Aires entre 1990 y 2003. Buenos Aires: Eudeba, 2010.

SCARZANELLA, Eugenia. Extranjeras en el país de Evita, la inmigración femenina italiana a Argentina (1946-1955). Anuario Americanista Europeo, n. 3, p. 145-171, 2005. Disponible en: <https://dialnet.unirioja.es/servlet/articulo?codigo=3160525>. Consulta: 06.02.2020.

SCOTT, Joan Wallach. Género e historia. México: Fondo de Cultura Económica, 2008. SILVA GARCÍA, Camila. Inmigración femenina y labores sanitarias y sociales en Chile de mediados del siglo XX. Nuevo Mundo Mundos Nuevos, octubre, 2019. Disponible en: < http://journals.openedition.org/nuevomundo/77132>. Consulta: 10.10.2019.

STRANGIO, Donatella. Emigrazione italiana "assistita" nel secondo dopoguerra. Popolazione e storia, v. 18, n. 2, p. 41-66, 2018. Disponible en: <https:// popolazioneestoria.it/article/view/894>. Consulta: 13.02.2020.

TAYLOR, Steve J.; BOGDAN, Robert. Introducción a los métodos cualitativos de investigación. Barcelona: Paidós, 1992.

WAINERMAN, Catalina. Mujeres que trabajan, Hechos e ideas. In: TORRADO, Susana (comp.). Población y bienestar en la Argentina del primero al segundo centenario: Una historia social del siglo XX. Tomo II. Buenos Aires: Edhasa, 2007, p. 325-352.

\section{Fuentes escritas}

CENTRO DE ESTUDIOS MIGRATORIOS LATINOAMERICANOS (CEMLA). Base de datos de arribo de inmigrantes. Buenos Aires, s./f.

PARROQUIA SAN JUAN BAUTISTA. Libro de Matrimonios. Valentín Alsina, 19261935.

PARROQUIA SAN JUAN BAUTISTA. Libro de Matrimonios. Valentín Alsina, 19461955.

\section{Fuentes orales}

CINTHYA SACCO. Entrevista realizada por Denise Ganza en el marco de la presente investigación. Valentín Alsina, 13 de diciembre de 2019. 
FAMILIA SESSA. Grupo focal con María Sessa, Elena Sessa, Giuseppa Sessa, Adelina Sessa y Ercilia Sessa, coordinado por Denise Ganza en el marco de la presente investigación. Valentín Alsina, 4 de junio de 2017.

MARÍA SESSA. Entrevista realizada por Denise Ganza en el marco de la presente investigación. Valentín Alsina, 27 de noviembre de 2019. 